\title{
Study of Factors Affecting the Adherence to HAART in HIV Infected Children
}

\author{
Ranju Choudhary ${ }^{1}$, Ravikant Nirankari² \\ ${ }^{1}$ Department of Dermatology, SMMH Medical College, Saharapur, CCU Meerut, Uttar Pradesh, India. \\ ${ }^{2}$ Department of Paediatrics, SMMH Medical College, Saharapur, CCU Meerut, Uttar Pradesh, India.
}

\section{ABSTRACT}

\section{BACKGROUND}

Adherence to medication, also known as compliance with medication, is the extent to which patients follow medical instruction.1,2 Adherence level of $90 \%$ or more is commonly cited as essential for HIV infected patients for maximum benefit of treatment. This level of adherence is also difficult to attain given that average adherence rate of $70-75 \%$ are found in primary care setting. ${ }^{3}$ We wanted to evaluate the factors that affect the adherence to highly active antiretroviral therapy (HAART) in children and counselling of attendant, parents, or caregivers for better adherence to HAART.

\section{METHODS}

The children who were HIV positive (confirmed by ELISA for HIV-I/or HIV-II) had been the part of this study. Follow up questionnaire method was followed in this study for total 30 patients who were enrolled in this study.

\section{RESULTS}

$>95 \%$ adherence level was found in $63 \%$ of patients in all six visits and all these patients had a better health status. Over all drug adherence level of $>95 \%$ in all age group was found in $60 \%$ of patients. Adolescent patients were found to be of fluctuating nature. $71 \%$ male patients found to be $>95 \%$ adherent while only $44 \%$ female patients were $>95 \%$ adherent to HAART. Some patients were found to be $100 \%$ adherent and multiple factors were present. Adherence program and counselling was the most important factor that increases adherence level after regular visits.
Corresponding Author: Dr. Ranju Choudhary, M.J.F. Marg, New Naveen Nagar, Saharanpur-247001, Uttar Pradesh, India.

E-mail: ranju.choudhary82@gmail.com

DOI: $10.14260 / j e m d s / 2022 / 80$

How to Cite This Article: Choudhary R, Nirankari R. Study of factors affecting the adherence to HAART in HIV infected children. J Evolution Med Dent Sci 2022;11(03):415-419, $10.14260 / \mathrm{jemds} / 2022 / 80$

Submission 01-02-2022,

Peer Review 15-02-2022,

Acceptance 18-02-2022,

Published 21-02-2022.

Copyright (C) 2022 Ranju Choudhary et al. This is an open access article distributed under Creative Commons Attribution License [Attribution 4.0 International (CC BY 4.0)]

\section{CONCLUSIONS}

Multiple factors affect the adherence to treatment and this is very difficult in children.

\section{KEY WORDS}

HIV, ART, ARV, HAART, Adherence, HIV Infected Children, STD. 


\section{BACKGROUND}

There are 355 ART centres and 725 link ART centres in India with a prevalence rate of $0.30 \%$, thousands of patients are taking ARV treatment from these centres among which a good proportion of patients are children. ${ }^{1}$ As far as adherence issue is concerned adherence to antiretroviral therapy is critical for HIV treatment success. Millions of children had lost one or both of their parents as a result of AIDS a sexually transmitted disease (STD). HIV infected children are facing a cycle of abuse, neglect, stigmatization, malnutrition, poverty and disease, have less chance of gaining an education and getting access to healthcare. There are two groups of children, first is infected (those who are HIV positive) and second is affected children (those who are member of an AIDS affected person's family). As far as adherence issue is concerned adherence to antiretroviral therapy is critical for HIV treatment success. Adherence level of $90 \%$ or more is commonly cited as essential for maximal viral suppression and immunological protection and in preventing the development of drug resistant viruses. ${ }^{4}$ This level of adherence is higher than that required for acceptable treatment of many other chronic diseases. Average adherence rate of $70-75 \%$ are found in most of the primary care setting in many studies.

To achieve optimal adherence to treatment in growing children, a thorough and detailed evaluation of the family environment, caretaker's attitudes, beliefs towards treatment and the ability to administer the prescribed regimen, needs to occur at the start of the treatment, and should be monitored at each visit during the entire treatment period. Any potential problems need to be resolved prior to treatment initiation. During early adolescence, a partnership with the child should be developed with the goal of providing disease education and empowerment regarding the need for medications and adherence. At this juncture, disclosure of HIV diagnosis to the child and to others in the child's environment becomes a key factor. ${ }^{5}$ A non-judgmental, trusting relationship between the teen and the care provider is crucial at this stage. In addition, the recognition and management of the HIV-associated myriad of psychosocial and mental health issues is another important facet, which has a major impact on adherence, and must be addressed for successful management of HIV. ${ }^{5}$

So, we wanted to evaluate the factors that affect the adherence to highly active antiretroviral therapy (HAART) in children and counselling of attendant, parents, or caregivers for better adherence to HAART.

\section{METHODS}

The children who were HIV positive (confirmed by ELISA for HIV-I/or HIV-II), registered and attending the ART centre OPD at SRN Hospital, Allahabad, associated with MLN Medical College, Allahabad had been part of this study. Study was conducted from March 2016 to Feb 2017.

Follow up questionnaire method was followed in this observational study. Attendants or care givers had been asked about 40 points about the drugs intake or missing the dose at every visit. Total six visits were evaluated. Patients were examined and asked for any adverse effects. The study was done on OPD basis. Randomly selected 30 patients were enrolled in this study, all of them were already taking ART from ART centre.

At every visit we evaluated the patients for adherence level. At first visit we took patients particulars, as name, age, sex, ART registration no, initial CD4 count, time of starting ARVs, and detailed family history.

A general physical examination was done in all patients, anthropometric assessments was done at every visit, we asked in detail of the following things.

- $\quad$ ART (Anti-Retroviral Therapy) pills count.

- Drugs and investigation record.

- Any change in drug schedule.

- History of missing doses

- Reasons of non-adherence

- Question about drug forms.

- Financial constraints.

- Institutional factors and quality of care at ART centre.

- Social support.

- Migration.

Calculation of adherence level by the following formula=

Total no.of pills taken as per schedule

$\overline{\text { Number of pills that should have been eaten by the patient till date }} \times 100$

\section{Statistical Analysis}

This is an observational study based on questionnaire method like a community survey with a random sample size of 30 patients only. So no other statistical equation is applied here.

\section{RESULTS}

Age distribution- we had no patient in less than 11-months age group, 2 patients were in 11-35-months age group, 3 patients were in 36-59-months age group, maximum no of patients $(80 \%)$ were more than 5 years of age in this study.

\begin{tabular}{|ccccc|}
\hline $\begin{array}{c}\text { Age } \\
\text { Group }\end{array}$ & $\begin{array}{c}\text { Total } \\
\text { Pts. }\end{array}$ & $\begin{array}{c}\mathbf{9 4 . 9 9 - 8 0 \%} \\
\text { Adherence }\end{array}$ & $\begin{array}{c}\mathbf{> 9 5 \%} \\
\text { Adherence }\end{array}$ & $\begin{array}{c}\text { \% Of Patients with }>\mathbf{9 5} \\
\% \text { Adherence }\end{array}$ \\
\hline 5 year & 5 & 2 & 3 & $60 \%$ \\
5-10 year & 15 & 6 & 9 & $60 \%$ \\
$>10$ year & 10 & 4 & 6 & $60 \%$ \\
\hline \multicolumn{4}{r}{ Table 1. Adherence Level According to Age Groups } \\
\hline
\end{tabular}

\begin{tabular}{|cccc|}
\hline Adherence Level & Male & Female & \% In Each Group \\
$>95 \%$ & 15 & 4 & 63 \\
$80-94.99 \%$ & 6 & 5 & 37 \\
$<79.99 \%$ & None & None & 0 \\
Total & $\mathbf{2 1}$ & $\mathbf{9}$ & \\
\hline \multicolumn{4}{c}{ Table 2. Total Adherence Level } \\
\hline
\end{tabular}

In a more simplified way, we distributed the patients in three groups.

- $\quad$ Group one $(<5$ years age $)-5$ patients

- Group two (5-10 years age) - 15 patients

- Group three ( $>10$ years age) -10 patients

- Sex distribution- Out of the 30 patients in this study, there were 21 male (70\%) and 9 female (30\%) patients.

- We took the optimum level of adherence $>95 \%$ in this study as cited by many studies already done on this. $(2,3)$ 
$<5$ years age group- there were total 5 pt. among them 3 patients had $>95 \%$ adherence level, similarly in 5-10 years age groups out of total 15 patients there were 9 pt. (60\%) with $>95 \%$ adherence level. Likewise, in $>10$ years age group there were total 10 patients and 6 patients $(60 \%)$ of them had $>95 \%$ adherence.

We found that 19 patients out of 30 (that is 63\%) were adherent to ART by $>95 \%$. Out of these 19 patients, 15 patients $(78 \%)$ were males and 4 patients, which is $22 \%$ of 19 , were females.

11 patients (37\%) of total 30 patients were between 80 $94.99 \%$ adherence, of whom 6 were male (54\%) and 5 were female patients (45\%).

We did not find any patient for $<79.99 \%$ total adherence level in last six visits.

\begin{tabular}{|ccc|}
\hline Adherence Level & CD4 Increase & CD4 Decrease \\
$>95 \%$ & $16(85 \%)$ & $3(15 \%)$ \\
$80-94.99 \%$ & 4 & 7 \\
$<79.99 \%$ & None & None \\
\multicolumn{3}{|c|}{ Table 3. Effect of Adherence on CD4 Level } \\
\hline \multicolumn{2}{|c}{} \\
\hline
\end{tabular}

In case of effects of adherence on CD4 status of patients, we observed that in patients who had $>95 \%$ adherence level 16 patients had a positive effect on CD 4 count in 6 months, around $85 \%$ of these 19 patients, while 3 patients $(15 \%)$ had decrease CD4 count in 6 month.

\section{Relation of Family History and Adherence to HAART}

We found that those patients who had a single parent alive on ART were 14 in total. 11patients (78\%) had $>95 \%$ adherence, while 3 patients had $80-94.99 \%$ adherence.

4 patients had both parents on ART and all these had $>95 \%$ adherence in last six month.

5 patients had both parents expired, in these 2 patients $(40 \%)$ had $>95 \%$ adherence and 3 patients $(60 \%)$ had 80 $94.99 \%$ of adherence level.

\begin{tabular}{|c|c|c|c|c|}
\hline Adherence & $\begin{array}{c}\text { Single Pare } \\
\text { on ART }\end{array}$ & $\begin{array}{l}\text { th Parents } \\
\text { on ART }\end{array}$ & $\begin{array}{l}\text { Both Parents } \\
\text { Expired }\end{array}$ & $\begin{array}{c}\text { Non-ART, NO } \\
\text { F/H OF HIV }\end{array}$ \\
\hline$>95$ & 11 & 4 & 2 & 3 \\
\hline $80-94.99 \%$ & 3 & None & 3 & 4 \\
\hline$<79.99 \%$ & None & None & None & None \\
\hline Total & 14 & 4 & 5 & 7 \\
\hline
\end{tabular}

Adherence to HAART in paediatric patients is very complex behaviour of patients, multiple factors affect the adherence level.

$>95 \%$ adherence level was found in $63 \%$ of patients in all six visits. And all these patients had a better health status and increased CD4 counts on follow up visits. We did not find any patients $<80 \%$ of total adherence level in our study.

- Age of the patients is very important factor to determine the adherence level in paediatric patients. Total parents' dependency for drug intake in younger age group make it clear that parents' education and positive motivation and counselling of parents is necessary to improve adherence in younger age group.

- Overall drug adherence level of $>95 \%$ in all age group was found in $60 \%$ of patients.
- Adolescent patients are of very fluctuating nature and they should be made aware of their HIV status and outcome of non-adherence, in our study we have seen that after proper counselling of these patients they were more regular in medicine intake and more aware of their health.

- $71 \%$ male patients found to be $>95 \%$ adherent while only $44 \%$ female patients were $>95 \%$ adherent to HAART. This may be due to prevailing social custom leading to more care of male child than female in India.

- Adherence level of $>95 \%$ or near $95 \%$ was found to be associated with positive effects on CD4 counts and improved health status of patients.

- $\quad$ Parents status affects the adherence level in many ways as-

- $\quad$ Those patients whose parents were on ART for their own illness had been found to be better adherent to HAART. Children with single parent on ART had >95\% adherence in $78 \%$ of cases. Children with both parents on ART had $>95 \%$ adherence in $100 \%$ of cases. In some families taking the medicine every day became a family behaviour and drug sharing was also found in these patients, when drug for children was finished parents were giving their own medicine to their children in half dose of adults and in this way, it improved the adherence in these patients.

- Orphan children had > $95 \%$ adherence level in only $40 \%$ of cases, while rest of the patients had $94.99-80 \%$ adherence level.

- $\quad$ Patients with no family history of HIV also had a >95\% adherence level, this was due to their family member's awareness and regular visits at ART centre.

- Some patients were found to be $100 \%$ adherent and multiple factors were there for this such as their age, parents ART status, siblings and care givers interest, effects of adherent program and counselling.

- Most of the patients missed the dose for two or three days because of their late visits and most important cause of late visits was financial constraints. Other cause of late visits were lack of care givers and loss of daily wages due to ART centre visit.

- Adherence program and counselling was the most important factor that increases adherence level after regular visits.

\section{DISCUSSION}

We found that adherence to HAART is more or less equal in all age groups. In present study, we found $60 \%$ patients had $>95 \%$ adherence level in each age group for six visits that is optimum according to many previous study,3,4 furthermore when we divided the patients in subgroups by age, then we found that patients in $<3$ years of age had better adherence to drugs. That may be due to their total dependency on parents for drug intake and if parents are motivated and well educated and counselled for adherence to HAART, they regularly come to ART centre and there is less chance of missing doses.

As the child grows up, problems develop with adherence level because of drugs taste, medicine forms and child's own 
interest and behaviour. All this play a major role in drug intake and non-adherence. ${ }^{6}$ In $>3$ years of age, many children refused to take medicine and sometimes pretend to take medicine but actually throw it out when parents don't supervise medicine intake. Furthermore, as the child grows up and know his/her own status of HIV positivity they become aware for their own health and became regular for medicine intake. In this study $>10$ years of age group, we had total 10 patients and we found most of them were aware about their HIV status and this increased their responsibility towards medicine intake. Patients of $>15$ years of age are more aware but often very fluctuating in nature that correlate with teens behaviour.

\section{Classification by Sex}

There were 21 male children and 9 female children in this study. Male patients had higher level of adherence to drugs, i.e., $71 \%$ male patients had $>95 \%$ adherence to the drugs while only $44 \%$ of female patients were found to be adherent for $>95 \%$ to drugs in total six visits. ${ }^{2}$

\section{Total Adherence Level}

We found that $63 \%$ of total patients were $>95 \%$ adherent to ART and $37 \%$ of total patients were $94.99-80 \%$ adherent to HAART. Prior to this study many studies defined optimal adherence as $>95 \% .4,7$ Adherence to drug is a dynamic nature of patients and it cannot be consistent every time.

\section{Effect of Adherence in CD4 Count}

Out of total 19 patients who were $>95 \%$ adherent, 16 patients had positive effect on CD4 count, i.e., $84 \%$ of total 19 patients had increase in total CD4 counts in last six month. ${ }^{8}$

\section{Family History and Adherence Level}

Family plays a major role in paediatric patients; many previous workers on this topic described the role of family. 9,10 All studies have mentioned about family responsibilities but very few described the relation between parents HIV status and children's medication. On the basis of family history, we divided the patients in 4 groups and tried to find out the relation between family's HIV status and drug intake in HIV infected children.

Group-1: Single parent on ART

Group-2: Both parents on ART

Group-3: Both parents expired

Group-4: Parents not on-ART/ No family history of HIV

GROUP 1-The cause of this increased adherence in this group may be the parent status of ART. As parents take medicine themselves, they also take care of their child for medicine intake and it becomes a family behaviour to take medicine every morning and evening and when parents come to ART centre, they bring their children to ART centre with them.

GROUP 2-There were total 4 patients in this group and all of them had $>95 \%$ adherence level to HAART. Here again, either mother or father are coming to ART centre for their own medication and they bring their children with them. Sharing of drugs between family members was found in this group. When medicine for the child was finished, parents were giving their own medicine to their child and this improved their drug intake. Compared to patients with single parent on ART where $78 \%$ patients had $>95 \%$ adherence level, $100 \%$ patients with both parents on ART had $>95 \%$ adherence. ${ }^{11}$

GROUP 3-Patients in this group had 94.99-80\% adherence level, in these cases siblings and grandparents were not very much interested and aware for child's health and drug intake and this decreased the adherence level. Grandparents' socioeconomic status and late visits also had been a factor for decreased adherence in this group. ${ }^{10}$ They were financially weak and could not come regularly for medicine to ART centre as it affects their daily income.

GROUP 4- Among 7 patients, 3 patients had $>95 \%$ adherence and 4 patients had $94.99-80 \%$ adherence level. In these 3 patients, 1 patient had no family history of HIV but family members and caregivers were very much aware about child's health. Age of the child was $>10$ year. This all affected the adherence in positive way. 4 patients had $94.99-80 \%$ adherence level in this group. Their parents were not on ART and could not come to ART centre regularly because of financial problems and they often missed doses due to lack of awareness of their parents. We found that parents were not much motivated in this group. ${ }^{12}$

\section{$100 \%$ Adherence Level (Full Adherence)}

Total 7 patients with $100 \%$ adherence to HAART makes $23.33 \%$ of total patients. These all had positive effect on CD4 count and improvement in health status. ${ }^{4,8} \mathrm{On}$ the basis of age as we found that there were $60 \%$ patients with $>95 \%$ adherence to HAART in every age group. Out of 7 patients 4 patients were 5-10 years of age. Out of these 4 patients, 3 had both parents on ART i.e., further a cause of good adherence to HAART in these patients. While 2 patients had one parent on ART. In this way we found that total 5 patients had either one or both parents taking medicine for their own HIV-AIDS and this makes $71 \%$ of these 7 patients and that seems significant. In rest of the two patients, one had no family history of HIV and another 1 patient had non-ART parents. But these both patients had a positively motivated family member and siblings who were interested in health of patients and they took care of patients and came to ART centre regularly.

\section{Facilitators of Adherence}

Adherence to any drug for chronic illness is very difficult when patient know that he has to take medicine for lifetime. Constant watch, adherent program and counselling are necessary to improve the adherence in paediatric patients. Regular counselling and involvement of patient directly in adherence program improves the adherence level in follow up and visits. ${ }^{13}$

\section{CONCLUSIONS}

Adherence to HAART in paediatric patients is a very complex behaviour of patients, multiple factors affect the adherence level. Making it a habit to take medicine every day is very 
difficult in children, especially when patients know that they have to take medicine for life long.

\section{REFERENCES}

[1] NACO Data on total HIV patients and on ART center in India.

[2] Shah CA. Adherence to highly active antiretroviral therapy (HAART) in pediatric patients infected with HIV: issues and interventions. Indian J Pediatr 2007;74(1):5560.

[3] UNGASS. India country progress report. March 1st 2008.

[4] Williams PL, Storm D, Montepiedra G, et al. Predictors of adherence to antiretroviral medications in children and adolescents with HIV infection. Pediatrics 2006;118(6):e1745-57.

[5] Shingadia D, Viani RM, Yogev R, et al. Gastrostomy tube insertion for improvement of adherence to highly active antiretroviral therapy in pediatric patients with human immunodeficiency virus. Pediatrics 2000;105(6):E80.

[6] Byrne M, Honing J, Jurgrau A, et al. Achieving adherence with antiretroviral medication for pediatric HIV disease. The AIDS Reader 2002;12(4):151-54, 161-4.

[7] Van Dyke RB, Lee S, Johnson GM, et al. Reported adherence as a determinant of response to highly active antiretroviral therapy in children who have human immunodeficiency virus infection. Pediatrics 2002;109(4);e61.

[8] Kumarsamy N, Venkatesh KK, Devaleenol B, et al. Safety, tolerability and effectiveness of generic haart in hivinfected children in South India. J Trop Pediatr 2009;55(3):155-9.

[9] Howland LC, Gortmaker SL, Mofenson LM, et al. Effects of negative life events on immune suppression in children and youth infected with human immunodeficiency virus type 1. Paediatrics 2000;106(3):540-6.

[10] Mukherjee JS, Ivers L, Leandre F, et al. Antiretroviral therapy in resource-poor settings. Decreasing barriers to access and promoting adherence. J Acquir Immune Defic Syndr 2006;43 Suppl 1: S123-6.

[11] Roberts KJ. Barrriers to antiretroviaral medication adherence in young HIV-infected children. Youth Society 2005;37(2):230-45.

[12] Catz SL, Kelly JA, Bogart LM, et al. Patterns correlates and barriers to medication adherence among persons ew treatments for HIV disease. Health Psychol 2000;19(2):124-33.

[13] Okuno J, Yanagi H, Tomura S. Is cognitive impairment a risk factor for poor compliance among Japanese elderly in the community? Eur J Clin Pharmacol 2001;57(8):58994. 